
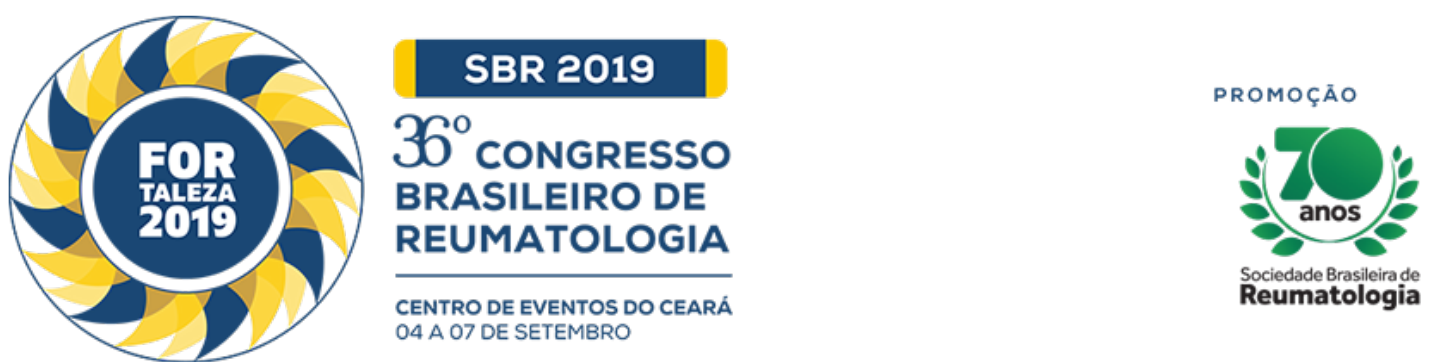

\title{
CENTRAL NERVOUS SYSTEM TUBERCULOSIS IN PATIENTS WITH SYSTEMIC LUPUS ERYTHEMATOSUS
}

Aloma Guinami Scabora (Universidade Estadual de Campinas, CAMPINAS, SP, Brasil), Alisson Aliel Vigano Pugliesi (Universidade Estadual de Campinas, CAMPINAS, SP, Brasil), Camille Constanza Codogno Postigo Castro (Universidade Estadual de Campinas, Campinas, SP, Brasil), Marília Bulhões Calheiros (Universidade Estadual de Campinas, Campinas, SP, Brasil), Vani Abreu de Souza Filho (Universidade Estadual de Campinas, Campinas, SP, Brasil), Amanda Pestana Braga (Universidade Estadual de Campinas, Campinas, SP, Brasil), Mariângela Ribeiro Resende (Universidade Estadual de Campinas, Campinas, SP, Brasil), Lilian Tereza Lavras Costallat (Universidade Estadual de Campinas, Campinas, SP, Brasil)

\section{BACKGROUND}

Tuberculosis (TB) is one of the world's most feared diseases due to its significant mortality rate. Involvement of central nervous system (CNS) occurs in $2-5 \%$ of patients. Generally, dissemination is hematogenous and clinical manifestations may mimic other conditions, infectious or not. Patients with systemic lupus erythematosus (SLE) are more likely to develop TB compared to the general population, and its symptoms may overlap with disease activity.

\section{CASE REPORT}

A 37-year-old female patient, diagnosed with SLE for 15 years, in use of hydroxychloroquine, started dry cough, chest pain, and dyspnea on moderate efforts in September 2018. She was treated for communityacquired pneumonia (CAP), but the cough persisted. Computed tomography (CT) revealed pulmonary nodules. She underwent bronchoscopy with bronchoalveolar lavage in November 2018, with negative results in studies and cultures for fungus and acid-fast bacilli (AFB). In December 2018, she started experiencing blurred vision, vertical diplopia and intense headache. She sought emergency care, in which an ophthalmologic cause was excluded and a magnetic resonance imaging of the skull was performed, demonstrating expansive cortical lesions in the CNS (Pictures 1 and 2). Cerebrospinal fluid (CSF) analysis was negative for $T B$, toxoplasmosis and cryptococcosis. Due to the persistence of cough, a sputum sample was requested, resulting positive in the direct search for AFB. Treatment for TB was started, with good response and progressive resolution of symptoms.

\section{CONCLUSION}

Although a small proportion of patients with TB manifest CNS disease, this condition, if not diagnosed and treated, may evolve with severe complications such as vasculitis, cerebral infarction, edema, and hydrocephalus. As the incidence is still high in our country and predisposition is greater in immunosuppressed patients, tuberculosis should always be considered as an important differential diagnosis of CNS manifestations. 